\title{
E-learning Tools and Remote Reconfigurable Systems for Engineering Education
}

\author{
Valery Sklyarov, Iouliia Skliarova \\ Department of Electronics, Telecommunications and Informatics, IEETA \\ University of Aveiro \\ Aveiro, Portugal \\ e-mail: sk1@ua.pt, iouliia@ua.pt
}

\begin{abstract}
This paper presents an original approach proposed and successfully used in University of Aveiro and targeted to advance e-learning tools and remote laboratories for state-ofthe-art engineering education. Nowadays, reconfigurable systems, in general, and FPGA (field-programmable gate array) based systems, in particular, constitute an essential part of engineering practice. The respective professional trends are, however, not reflected accordingly in contemporary engineering education curricula. The paper argues importance of reconfigurable systems in education and proposes effective learning methods and tools, which include laboratory templates, animated tutorials, education-oriented examples, a remotely reconfigurable prototyping system, and a virtual software/reconfigurable hardware co-simulation environment.
\end{abstract}

Keywords-E-learning; remote reconfiguration; prototyping systems; engineering education

\section{INTRODUCTION}

Tremendous progress in the scope of field-programmable gate array (FPGA) technology has made it possible to advance configurable microchips from simple gate arrays that appeared on the market in the mid-1980s to multiplatform FPGAs containing more than 10 million system gates and targeted to the design of very complicated engineering systems. Developing engineering systems on the basis of high capacity FPGAs involves vast variety of design tools and requires a large number of well-prepared engineers in the relevant areas [1]. Hence new trends must be appropriately reflected in the respective pedagogical activity and this strongly demands an ongoing review of university curricula.

Developing digital systems on the basis of high capacity FPGAs requires the extensive use of computer-aided design (CAD) tools. In fact the electronic design automation business has profoundly influenced the integrated circuit business and vice-versa [2]. Traditionally, FPGA-targeted CAD systems support schematic and hardware description language-based design flows involving model-specific tools (such as synthesis of finite state machines from a graphical specification) and IP (intellectual property) core generators based on parameterization or templates. Recently, commercial CAD tools allowing digital circuits to be synthesized from system level specification languages (such as Handel-C and SystemC) as well as high-level programming languages (such as $\mathrm{C}++$ ) have appeared on the market. Thus, the domain of reconfigurable systems design is very dynamic and many-sided. Evidently an ongoing review of universities' curricula is necessary in order to incorporate the recent advances in FPGA architectures, design methods, and CAD tools. In other words, the curriculum must be sensitive to changes in technology and new developments in pedagogy and should emphasize the importance of lifelong learning [3]. Significant assistance in this direction can be provided through the extensive use of elearning and advanced laboratorial tools [4]. It is important to note that on the one hand the mentioned tools are very helpful for teaching disciplines concerned with FPGA-based design and on the other hand reconfigurable systems themselves provide a very significant basis for the development of advanced laboratorial systems that can be used for vast varieties of fundamental disciplines in engineering education.

The remainder of the paper is organized in six sections. Section II presents further arguments to the importance of reconfigurable systems in engineering education. Section III describes the proposed e-learning tools. Section IV characterizes the developed remotely reconfigurable prototyping system. Section V discusses a virtual visual environment for engineering education. Section VI summarizes the contributions of the paper. The conclusion is given in Section VII.

\section{IMPORTANCE OF RECONFIGURABLE SYSTEMS FOR ENGINEERING EDUCATION}

Reconfigurable systems possess a number of particularities both in implemented architectures and in functional capabilities. The most important of them are:

- Configurability that makes it possible to consider such systems as soft application-specific integrated circuits, i.e. soft ASICs.

- Reconfigurable devices are hardware circuits that can be designed, physically implemented and tested remotely.

- They introduce a new distinguishing computing paradigm and eliminate the necessity for traditional von Neumann architecture.

- Parallelism becomes the most important technique allowing system performance to be increased.

- Reconfigurable systems open practically unlimited opportunities for design space exploration and comparison of alternative and competitive solutions. 
- They permit any required external interface to be easily established.

- $\quad$ Systems can be composed of novel and pre-designed components and also temporarily embedded circuits for testing and verification. This significantly simplifies the design process and increases importance of such tools as libraries, templates, IPcores, and embedded testers.

As a rule, traditional education does not provide sufficient knowledge in the areas listed above. In other words, students after graduating from universities are not capable to apply their engineering acquirements to the design of well structured and optimally organized reconfigurable systems. Due to the great significance of reconfigurable systems for many industrial areas, tending to be significantly increased in the future, the importance of the relevant topics in education is evident. Besides of teaching reconfigurable systems themselves, it is very valuable to involve reconfiguration in engineering curricula, which would permit to improve education in vast varieties of disciplines and we would like to give just a few examples:

- Using reconfigurable devices for laboratory works in such disciplines as digital electronics, digital design, computer architectures, etc. enables the students to implement and verify in hardware numerous circuits on the fly.

- Using reconfigurable devices for engineering projects, on the one hand, significantly shortens the design process and, on the other hand, makes it possible to construct and test physical hardware remotely.

- Reconfigurable systems enable instructors of various disciplines to realize evolutionary strategy in education. Indeed, since not only the complete projects but also project components are reusable and, as a rule, parameterizable, the future student projects and laboratory works can be partially based on reusable design units developed by the previous generations of the students. Thus, evolutionary strategy can be applied directly, i.e. experience of current students will be integrated in the experience of future students. This strategy, in particular, forces the students to document their designs properly.

An efficient educational process in the considered scope has to combine real hand-on experiments with FPGA-based boards, simulation, and tests in a remote/virtual laboratory (in accordance with suggestions done in [5] for different domains, including engineering, natural sciences, education, and psychology). Remote and virtual laboratories have gained popularity in engineering education since they allow alleviating the problems of limited accessibility and high running costs of real laboratories [6].

From the four application areas of remote laboratories identified in [7], we envision two possible usages:

- "localized" remote laboratory, which can be accessed by students through the Internet to perform experiments outside of class hours. The main objective is to remove fixed time limits and to give more time to students to familiarize themselves with past and future projects.

- "distant" remote laboratory, which can be used in distance education.

We would like to underline that the developed tools are not intended to replace the real hand-on experience of students; instead they serve to complement and enrich the real laboratory projects.

The majority of available electrical engineering remote laboratories deal with fixed experiments [6-8], where the students are only able to control a limited number of parameters and to observe visually behavior of a system and/or to receive the resulting measurement data for subsequent processing and analysis. Contrariwise, the remote laboratory described in this paper does not limit in any way the type of circuit/system to be implemented. This is possible thanks to FPGA inherent reconfigurability. A similar FPGAbased remote laboratory is considered in [9]. However, comparing to [9], the proposed here tools have a number of distinctive features that will be described in detail in the subsequent three sections.

\section{E-LEARNING TOOLS}

The developed e-learning tools are organized as a set of laboratory templates; tutorials, education-oriented examples and supplementary materials.

A laboratory template is a Web page, which enables the students to select properly all the required components of a project and additional materials that might be useful.

Tutorials are divided into the following groups:

- Design scenarios;

- Specification, synthesis and implementation of FPGA-based circuits from hardware description languages;

- Standard interfaces with typical peripheral devices;

- Design methods targeted to reconfigurable systems.

Education-oriented examples demonstrate how to construct reconfigurable circuits based on:

- Reusable hardware description language fragments (RHDLF);

- Templates;

- Demonstration resources organized as educationtargeted libraries.

RHDLFs are pieces of easily customized hardware description language (HDL) code that can be inserted into more complicated HDL-based projects. For example, RHDLFs might be specifications of typical operations, such as interface with a keyboard or binary to BCD converters.

Templates are complete customizable specifications for such circuits as finite state machines (FSMs), hierarchical FSMs, parallel FSMs, etc.

Demonstration resources are composed of software programs (that enable the students to understand easier and better the proposed task), similar projects and scenarios (illustrating the required sequence of steps and the expectable output or results). The primary objective of education-oriented examples is to explain how to develop real-world projects composed of design cores worked out by 
the students and supplied components simplifying the project and shortening the development lead-time. The examples are organized in such a way that enables teachers to provide sufficient knowledge within the limited time.

Basically, the students' projects are divided in core and supplementary components. The boundary between these components is fuzzy. Dependently on educational targets we can provide either more or less supplementary components and this makes it easier to adapt the proposed technique to different university curricula. In particular, in the University of Aveiro, reconfigurable systems are taught to $4^{\text {th }}$ year students of computer engineering curriculum and $5^{\text {th }}$ year students of electronics and telecommunications curriculum. The background of these two groups of students is different but what is important is that independently of the actual deepness of the required basic knowledge, the students can work with real-world examples. The available e-learning tools provide significant assistance making it possible to complete real-world projects within the limited time normally available for laboratory classes.

\section{RECONFIGURABLE SYSTEMS WITH WIRED AND WIRELESS INTERACTIONS}

The majority of laboratory classes assume several steps that have to be completed by the students. The last of them is implementation, verification and demonstration of the project in FPGA. An FPGA-based prototyping system (see Fig. 1) has been developed for such purposes.

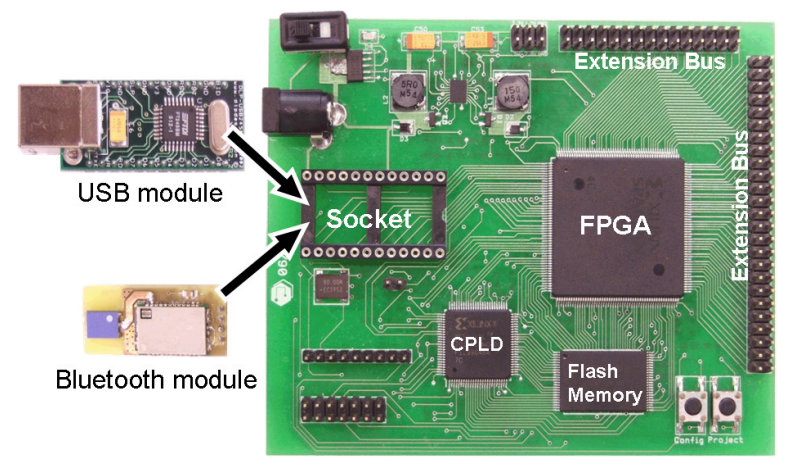

Figure 1. The developed prototyping board with wired and wireless interfaces.

In general, the developed system possesses the following primary distinctive features:

- The core FPGA can be configured using wired (USB) and wireless (Bluetooth) interfaces;

- The developed software/hardware components provide support for: 1) wired reconfiguration/interaction through USB interface; 2) autonomous reconfiguration from onboard flash memory; and 3) remote wireless reconfiguration/interaction through Bluetooth interface;

- The design process is supported by numerous developed tools, such as templates, design libraries,
IP cores, etc. Many of them are oriented to remote control and reconfigurability;

- The remote interaction capabilities are supported by the developed software making it possible to verify different projects virtually and remotely in such a way that FPGA-based hardware is considered to be a remotely accessible component and the required functionality is implemented both in physical hardware (FPGA-based prototyping board) and in software supporting visual interface for virtual verification of the developed system (this feature will be considered in the next section in more detail).

It is known that there are many prototyping boards available. Why one more board has been designed? First of all, the board was planned to be used by undergraduate and postgraduate students of electronics, telecommunications and computer engineering curricula. These students have to acquire profound knowledge and abundant experience in the scope of electronic circuits design and software engineering. Therefore, we would like to use open-source hardware/software tools which are completely understandable without any hidden feature. Besides, such tools have to satisfy all necessary functional requirements. The most appropriate solution was to develop the board in the department by postgraduate students, which can easily spread the required knowledge and experience to other students. It was done and the following benefits have been obtained:

1) The board has become an ideal platform for the development of both new electronic devices and software. Indeed it does not have any hidden or unknown element or source code. Such open hardware and software is very uncommon for commercial prototyping systems, i.e. source code for FPGA configuration, communication with host computers, etc. is usually not provided.

2) The board has become a hardware core that is being continuously improved by the students. In other words, the current student generation makes the board better for subsequent student generations. For example, many hardware extensions (depicted and characterized in Fig. 2) were designed by the students.

3) The board is an ideal element for remotely controlled embedded systems, mainly because it supports wireless interface. The latter is not widely available for FPGA-based prototyping systems. Moreover, at the beginning of the development our board was the only available FPGA-based prototyping board supporting wireless reconfiguration and interaction.

4) The board is a very flexible and easily extendable core [10], which can be customized for many practical applications in such a way that the developed board-based system will include only the required components. This feature is also not so common for the majority of commercially available boards, which contain many auxiliary devices that are not required for particular user applications. 


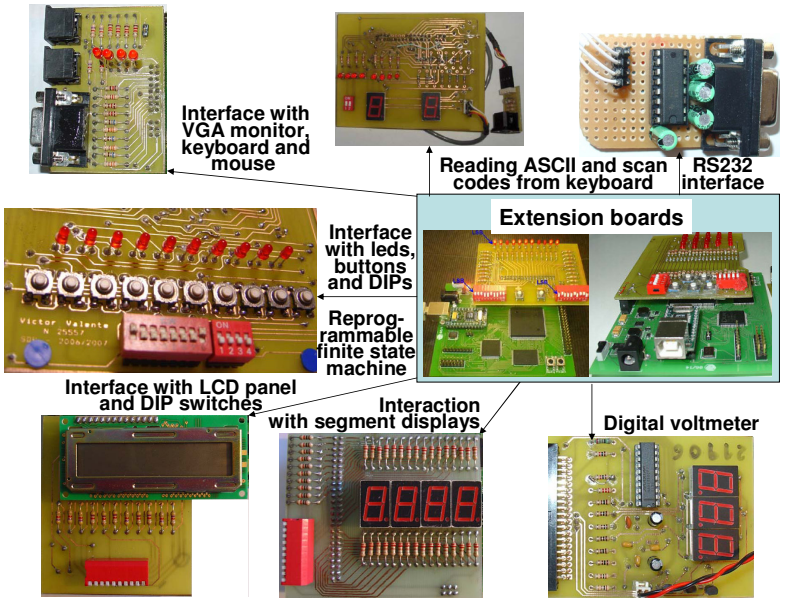

Figure 2. Examples of extension boards developed by the students.

\section{VIRTUAL VISUAL ENVIRONMENT FOR ENGINEERING EDUCATION}

Fig. 3 demonstrates the basic idea of a virtual visual environment. As a rule, circuits implemented in FPGAs are parts of larger circuits making up the designed system. The latter might be composed of other circuits, sensors, actuators, electromechanical devices, etc. In general, the virtual visual environment consists of the following three primary parts (see Fig. 3):

1) Virtual devices implemented in a host computer. They are virtual devices because they are implemented in software and provide such functionality that is very similar to physical devices. They are visual because we are able to observe the functionality (such as different motions of mechanical elements, states of electronic components, etc.) in visual mode on a monitor screen (or possibly in some other connected peripheral devices). They are easily controllable because we can carry out numerous functional and timing scenarios, for example, test just the selected fragments of implemented algorithms, execute algorithmic steps faster or slower, etc.

2) Physical devices implemented in FPGA and interacting with virtual devices in such a way that allows to make up the designed system, i.e.: physical devices + virtual devices $=$ the designed system. Such system is flexible and extendable, because functionality of both software and reconfigurable hardware can be altered.

3) Hidden from the end users software/reconfigurable hardware interface providing interaction between the virtual and physical devices.

An example is given in the upper part of Fig. 3 where an FPGA on the right-hand side controls an assembly line on the left-hand side and the respective interaction is established through an FPGA/host computer interface. Another example can be taken from the area of embedded systems [11]. Suppose it is necessary to design a device controlled by a sequence of instructions such as "switch on/off", "set in a given state", "reset", etc. These instructions affect the behavior of an execution unit via actuators, and the sequence of the instructions depends on the states of the execution unit sensors. The actuators and sensors can be electronic, optical, mechanical, etc. The proposed technique permits:

- To verify the embedded system entirely in software;

- To implement the embedded system partially in software and partially in hardware;

- To carry out hardware/software co-simulation with fuzzy boundary between hardware and software (i.e. to analyze the embedded systems with either more software and less hardware or vice versa).

This task is very interesting and useful for students dealing with different problems from vast variety of application areas, such as:

- The mentioned above assembly lines (see upper part of Fig. 3) and embedded systems;

- Problem-oriented computations, such as solving combinatorial search problems. In this particular case, the execution unit of the considered processing block can be modeled in software and the control algorithms can be implemented in hardware;

- Design of electromechanical peripheral devices, such as plotters (see examples in [11]). In this case electromechanical part can be modeled in software and electronic (logic) part can be implemented in hardware.

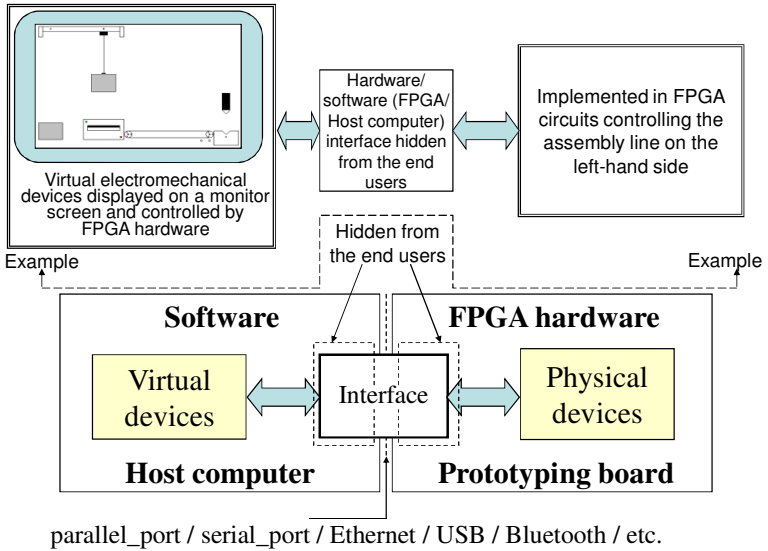

Figure 3. Visual virtual environment with an example.

It is important that the virtual visual environment makes it possible to create vast variety of virtual peripheral devices for the FPGA-based prototyping core considered in section IV. Indeed, the presented above basic idea of laboratory works allowing real-world projects to be implemented, is the division of the project into core components, developed by the students, and supplied components, given to the students. The latter are mainly interface circuits for peripheral devices providing data input and output. The proposed technique allows implementing all such devices virtually in a way, shown in Fig. 4.

The system depicted in Fig. 4 displays virtual peripheral devices (namely pushbuttons, an LCD panel, a segment display, and LEDs) and communications with such devices are organized much like communications with physical peripheral devices 


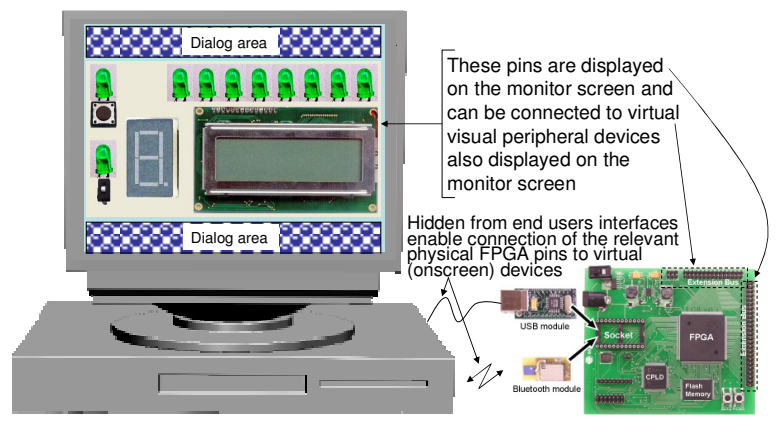

Figure 4. Prototyping system with FPGA and virtual peripheral devices.

\section{PRACTICAL CONTRIBUTION}

In the scope of e-learning tools all the elements described in section III were implemented and tested at different levels within the disciplines on reconfigurable computing and reconfigurable digital systems for students of electronics, telecommunications and computers curricula. All the elements are being used in pedagogical practice and they are available through the Internet $[12,13]$.

The reconfigurable system (considered in section IV) was entirely implemented and tested. The core board contains the XC3S400 FPGA of Xilinx's Spartan-3 family (more details about the board can be found in [10]). The system was employed in pedagogical practice starting from 2006/2007. There are many extension boards such as that are shown in Fig. 2 designed, implemented and tested by the students.

The virtual visual environment was developed starting from 2000. The first results in this area are presented in [11]. Many software/reconfigurable hardware projects have been proposed to the students. At the moment, the prototyping system with virtual peripheral devices (see Fig. 4) was tested. It functions well but some additional time is required to introduce this environment to pedagogical practice. Distant interface between the FPGA-based board and user computers (established through the Internet) was also implemented and verified. Both wired and wireless Internet connections and also both wired (USB) and wireless (Bluetooth) interactions between the board and the server computer have been tested. The server computer was used just to control access to the board through the Internet.

\section{CONCLUSION}

The paper argues the importance of reconfigurable systems in engineering education and describes the developed e-learning tools, the prototyping system, and the virtual visual environment that make it possible to increase the efficiency and productiveness of teaching in this area. Elearning tools are organized as a set of laboratory templates, tutorials, education-oriented examples, and supplementary materials. All these tools are available online through the Internet. The FPGA-based prototyping system was entirely designed, implemented and tested at the department. Very significant assistance was provided by the students and they designed numerous extension boards that are going to be used for future generations of students. This establishes, in particular, an evolutionary strategy enabling the future students to use the improved and extended devices/tools created by the previous students. Finally, the developed virtual visual environment provides significant assistance for design space exploration and hardware/software cosimulation. This environment can be very efficiently employed in remote laboratories for engineering education.

In 2009 our project "Use of HP Mobile Technology to Enhance Teaching Reconfigurable Systems for Electrical and Computer Engineering Curricula" won a HewlettPackard award within the "2009 HP Innovations in Education" initiative [14]. We believe that the equipment and supporting tools received within this project will allow to further increase the efficiency, effectiveness, and quality of reconfigurable systems education making it possible to reduce the gap between the industrial requirements and capabilities of engineering training.

\section{REFERENCES}

[1] V. Sklyarov, I. Skliarova, "Teaching Reconfigurable Systems: Methods, Tools, Tutorials, and Projects", IEEE Trans. on Education, vol. 48, no. 2, 2005, pp. 290-300.

[2] D. MacMillen, M. Butts, R. Camposano, D. Hill, T. W. Williams, "An Industrial View of Electronic Design Automation", IEEE Trans. on Computer Aided Design of Integrated Circuits and Systems, vol. 19, no. 12, Dec. 2000, pp. 1428-1448.

[3] "A Vision for ECE Education in 2013 and Beyond", IEEE Trans. on Education, vol. 46, no. 4, November 2003.

[4] R. Marín, P. J. Sanz, P. Nebot, R. Wirz, "A Multimodal Interface to Control a Robot Arm via the Web: A Case Study on Remote Programming", IEEE Trans. on Industrial Electronics, vol. 52, no. 6, Dec. 2005, pp. 1506-1520.

[5] J. Ma, J. V. Nickerson, "Hand-On, simulated, and remote laboratories: A comparative literature review", ACM Computing Surveys, vol. 38, no. 3, Article 7, Sept. 2006.

[6] Z. Nedic, J. Machotka, "Remote Laboratory NetLab for Effective Teaching of $1^{\text {st }}$ Year Engineering Students", iJOE International Journal of Online Engineering, vol. 3, no. 3, Article 8, 2007.

[7] D. Z. Deniz, A. Bulancak, G. Özcan, "A Novel Approach to Remote Laboratories", in Proc. 33 ${ }^{\text {rd }}$ ASEE/IEEE Frontiers in Education Conference, CO, USA, November 2003, vol. 1, pp. T3E-8 - T3E-12.

[8] D. Hercog, B. Gergic, S. Uran, K. Jezernik, "A DSP-based Remote Control Laboratory", IEEE Trans. on Industrial Electronics, vol. 54, no. 6, Dec. 2007, pp. 3057-3068.

[9] L. S. Indrusiak, M. Glesner, R. Reis, "On the Evolution of Remote Labs for Prototyping Digital Electronic Systems", IEEE Trans. on Industrial Electronics, vol. 54, no. 6, Dec. 2007, pp. 3069-3077.

[10] M. Almeida, B. Pimentel, V. Sklyarov, I. Skliarova, "Design Tools for Rapid Prototyping of Embedded Controllers", Proc. $3^{\text {rd }}$ International Conference on Autonomous Robots and Agents ICARA'2006, Palmerston North, New Zealand, December 2006, pp. 683-688.

[11] V. Sklyarov, "Hardware/Software Modeling of FPGA-based Systems", Parallel Algorithms and Applications, vol. 17, no. 1, 2002, pp. 19-39.

[12] V. Skliarov, [Online]. Available: http://www.ieeta.pt/ skl/

[13] I. Skliarova, [Online]. Available: http://www.ieeta.pt/ iouliia/.

[14] HP project "Use of HP Mobile Technology to Enhance Teaching Reconfigurable Systems for Electrical and Computer Engineering Curricula", [Online]. Available: http://www.ieeta.pt/ skl/HP_project.html. 